\title{
Doblando y desdoblando la trama urbana en la India contemporánea
}

\author{
Sergio Román Aliste \\ Universidad Complutense de Madrid, \\ Departamento de Historia del Arte III (Contemporáneo)
}

\begin{abstract}
RESUMEN
Las metáforas del plegamiento, y a veces la acción física de doblar (o desdoblar), permiten vislumbrar, comprender e incluso plantear alternativas y soluciones a muchos de los problemas del urbanismo indio contemporáneo. Este artículo se centra en algunas obras artísticas y proyectos que giran en torno a esa idea, tomando como punto de partida la ciudad de Bombay.
\end{abstract}

Palabras clave: Urbanismo; crecimiento orgánico; altas densidades; Charles Correa; Rahul Mehrotra.

Folding and unfolding the urban grid in contemporary India

\begin{abstract}
Metaphors of folding, and sometimes the physical action of folding (or unfolding), show alternatives and can propose solutions to many of the problems of contemporary Indian urbanism. This paper will focus on some artworks and projects around that idea, taking Mumbai as starting point.
\end{abstract}

Keywords: Urbanism; organic growth; high densities; Charles Correa; Rahul Mehrotra.

" $i$ Y hay tantas historias que contar, demasiadas, tal exceso de vidas acontecimientos milagros lugares rumores entrelazados, una mescolanza tan densa de lo improbable y lo mundano!"

Salman Rushdie, Hijos de la medianoche

Una ciudad donde conviven varias formas de urbanismo en un mismo espacio físico, como un gran tejido compuesto de diferentes tipos de tramas, que al doblarse densifica su textura de modo siempre impredecible. Podríamos referirnos con este símil a cualquiera de las ciudades indias más populosas a principios del siglo XXI (o incluso a otras grandes metrópolis de Asia, África o Latinoamérica), aunque es Bombay (hoy conocida como Mumbai) la que se ajusta más a los términos de densificación, y la más apropiada por la importancia de la industria textil en su desarrollo urbano. 
El centro metropolitano de Bombay, formalmente una isla, es el resultado de procesos artificiales de alisado del archipiélago que la formaba originalmente, antes de que los imperios coloniales, portugués primero y británico después, actuaran sobre él ganando terreno al mar. Pronto Bombay se convirtió en el principal puerto de la Costa Malabar, y no tardó en desbancar a Calcuta como principal centro comercial e industrial de la India Británica, especialmente tras la inauguración del Canal de Suez (1869) y la implantación hacia mediados del XIX de una red de fábricas textiles cerca de los muelles de la bahía. La primacía de Bombay como centro de las manufacturas textiles se prolongó más allá de la Independencia (1947), hasta que las huelgas prolongadas que paralizaron el corazón productivo de la ciudad desde 1982 crearon un gran vacío en el mismo núcleo de la vieja urbe colonial, obligando a una reconversión en la que aún se encuentra inmersa, al mismo tiempo que la población de la metrópoli aumentaba de manera exponencial - lo ha venido haciendo desde la década de 1940 sin cesar -, llegando desde los años sesenta a unos límites insostenibles, que se han visto superados, no obstante, con creces. La ausencia de actividad en los telares del corazón de la trama urbana de Bombay ha coincidido, por tanto, con la densificación humana más brutal que podríamos imaginar.

El arquitecto indio Rahul Mehrotra (Nueva Delhi, 1959) ha seguido la estela de otros planificadores como Charles Correa (Hyderabad, 1930) en su preocupación por las condiciones urbanas de la ciudad de Bombay, aportando tanto soporte teórico como alternativas tangibles. A Mehrotra pertenece la idea de ciudad como medio elástico, aquél que resulta no de una gran visión, sino de un gran ajuste ${ }^{1}$. En este punto es donde hemos de entender la convivencia de varios modos urbanos en un mismo entorno espacial: Rahul Mehrotra habla de dos ciudades que se superponen diariamente en Bombay: estática y cinética; formal e informal, que no constituyen entidades polares, sino imbricadas. La primera depende de la arquitectura para su representación y se expresa en materiales perdurables; es fundamentalmente bidimensional, aunque la perspectiva de observación es clave para su comprensión. La segunda, por su parte, constituye un flujo permanente que se rige por la dimensión temporal y los mecanismos cíclicos a través del uso de estructuras efímeras, materiales de desecho, y por la pura organicidad en su desenvoltura a través del entramado formal o estático. En la Ciudad Cinética la perspectiva no cuenta; los ritmos del medio urbano rodean al transeúnte en una permanente reinvención de la metrópoli: "los lugares, en la India, no se poseen, se hacen. Se hacen entre todos, con el movimiento, acompasando el cuerpo" ${ }^{2}$. En este escenario de construcción comunitaria, "las ciudades en India han resultado lugares críticos para la negociación entre la

1 MEHROTRA, Rahul, "Negotiating the Static and the Kinetic: The emergent urbanism of Mumbai", en HUYSSEN, Andreas (ed.), Other cities, other worlds: Urban imaginaries in a Globalizing Age, Durham / London, Duke University Press, 2008, p. 206.

2 MAILLARD, Chantal, "El espacio sonoro de la India", en Contra el arte y otras imposturas, Valencia, Pre-textos, 2009, p. 246. 
élite y las culturas subalternas [...]. La fragmentación de los emplazamientos de producción y servicios ha derivado en un nuevo tipo de urbanismo a modo de bazar, que ha tejido su presencia a través del paisaje urbano en su conjunto" ${ }^{3}$.

La característica cinética de la ciudad se proyecta, de este modo, más allá de la mera noción de movimiento, como una colonización transitoria - pero reiteradadel entramado urbano a través de estructuras (puestos de venta ambulante, mercadillos, templetes efímeros o pandals, etc.) o acontecimientos como bodas o festivales religiosos, que lo transforman continuamente. Como se ha dicho, "la calle es el espacio primario de experiencia en una ciudad como Bombay. Funcionando no sólo como vías de conexión entre lugares, las calles de Bombay constituyen el espacio por excelencia de lo social"'4. Sin embargo, lo que aquí nos interesa no tiene que ver específicamente con los procesos políticos o sociales que genera la actividad de una ciudad de estas características, ni siquiera a través de su reflejo en el arte, sino con el modo en que la ciudad experimenta los fenómenos de condensación o densificación, tanto desde el punto de vista físico como desde de la vivencia o experiencia.

El crítico Ranjit Hoskote ha definido al que quizás es el pintor por excelencia de la ciudad de Bombay, Sudhir Patwardhan (Pune, 1949), como el observador cómplice, aquél que se debate entre la intimidad y la distancia de la escena que representa, como se puede apreciar en la obra titulada $\operatorname{Town}^{5}$ (1984, óleo sobre lienzo, 185 x $154 \mathrm{~cm}$, Peabody Essex Museum). Al hilo del tipo de artista que encarna Patwardhan para la ciudad en la que vive y con la que convive ${ }^{6}$, se ha dicho que "en el momento de la identificación con un protagonista subalterno, [ese creador] puede señalar cada faceta de la vida moderna en la ciudad"7. Podríamos ver a Patwardhan como Baudelaire al Sr. G., como un curioso de las multitudes, tal como Edgar Allan Poe describía al protagonista de su cuento El hombre de la multitud, que el poeta francés citaba con entusiasmo ${ }^{8}$. Sin embargo, aquí la relación con la muchedumbre probablemente avance en dirección contraria, dado que en Bombay,

3 MEHROTRA, Rahul (2008), op. cit., pp. 205-207.

4 ZITZEWITZ, Karin, "The Moral Economy of the Street. The Bombay Paintings of Gieve Patel and Sudhir Patwardhan", Third Text, vol. 23, n 2, 2009, p. 151.

5 HOSKOTE, Ranjit, Sudhir Patwardhan. The complicit observer, Mumbai, Popular Prakashan, 2010, p.18

6 Existe aquí un cambio importante con respecto a la mirada de muchos artistas de la inmediata postindependencia, que llegaban a Bombay desde zonas más o menos rurales, y proyectaban su modo de ver "extrañado", percibiendo a la ciudad como "una alienante estructura laberíntica" o algo ajeno a su propio ser, como se puede observar en el dibujo de David Newton Souza titulado Portrait and Township (1957, tinta sobre papel, 20,3 x 32,4 cm, Delhi Art Gallery). Ver KARODE, Roobina y SAWANT, Shukla, "City Lights, City Limits. Multiple metaphors in everyday urbanism, en SINHA, Gayatri, Art and Visual Culture in India. 1857-2007, Mumbai, Marg Publications, 2009, pp. 192-193.

7 KAPUR, Geeta y RAJADHYAKSHA, Ashish, "Bombay/Mumbai 1992-2001", en BLAZWICK, Iwona (ed.), Century City. Art and Culture in the Modern Metropolis, catálogo de la exposición, London, TATE, 2001, p. 22.

8 BAUDELAIRE, Charles, El pintor de la vida moderna (ed. de Antonio Pizza y Daniel Aragó), Murcia, Colegio Oficial de Aparejadores y Arquitectos Técnicos, 1995 (1868), p. 84. 
con frecuencia (como explica gráficamente Suketu Mehta en su crónica personal sobre la ciudad ${ }^{9}$ ) se huye del gentío en busca de una soledad inalcanzable. Y aunque en determinados momentos de su trayectoria Sudhir Patwardhan haya representado con insistencia figuras solitarias en espacios abiertos o cerrados, otras obras clásicas de su producción como la mencionada Town, muestran una densificación activamente buscada por el artista, pero no precisamente la que podríamos obtener tomando una instantánea única de la calle. Gieve Patel, artista muy próximo a Patwardhan, escribió lo siguiente acerca de su modo de pintar:

"En la obra titulada Town, Sudhir Patwardhan aplica el conocido procedimiento de observar la ciudad desde una pluralidad de ángulos para luego consolidarlos en una sola imagen. De este modo, el ámbito de visión es mucho mayor que el que se deriva de las dimensiones del lienzo. Aquí está la diferencia. Tiene extremo cuidado para evitar cualquier sensación molesta de compresión o distorsión óptica. Nos conduce a creer que la escena condensada y sosegada que se presenta en el lienzo es lo que veríamos de modo normal fuera de ella, en cualquier momento con nuestros propios ojos. Éste es el artificio de la obra, un recurso que nos facilita una entrada plácida en una de nuestras desapacibles ciudades" 10

El arquitecto Charles Correa ha dedicado algunas páginas a estudiar esas sensaciones con las que se convive en una ciudad como Bombay dentro del texto Great City... Terrible Place, incluido en el estudio titulado The New Landscape, que puede ser considerado un auténtico manifiesto sobre las condiciones necesarias para el desarrollo de un nuevo paisaje urbano en India. Una paradoja se cierne sobre la ciudad de Bombay, que Correa ha sabido ver, y es que el altísimo grado de actividad, de energía y de vivencias resulta fundamental para su desarrollo como una de las grandes metrópolis contemporáneas, al mismo tiempo que los desajustes en su entorno físico (provocados por las desigualdades y exorbitantes densidades de población en una espacio geográfico muy limitado) la convierten en un lugar insostenible.

La organicidad del medio urbano indio suele prestarse a la utilización de un lenguaje biológico para la ilustración de sus estructuras internas y funcionamientos ${ }^{11}$. Sin embargo, Charles Correa ha dado un paso más al acudir a teorías alternativas en la historia del pensamiento científico para tratar de explicar las razones de esa condensación humana, para lo que echa mano de las ideas del paleontólogo y jesuita francés Pierre Teilhard de Chardin (1881-1955):

9 MEHTA, Suketu, Ciudad total. Bombay perdida y encontrada, Barcelona, Mondadori, 2006 (2004), p. 163.

10 PATEL, Gieve, (texto sin título sobre S. Patwardhan, Sunday Observer, Bombay, 30 de diciembre de 1984), cit. en Sudhir Patwardhan, catálogo de exposición, París, Centre Georges Pompidou, 1986, p. 15.

11 Ver el análisis comparativo entre el modelo urbano tradicional en el norte de India y los prototipos de urbanizaciones implantados desde la colonización británica y que hoy son propios de las clases medias-altas: PRASAD, Sunand, "A Tale of Two Cities: House and Town in India Today”, en TILLOTSON, G.H.R. (ed.), Paradigms of Indian Architecture. Space and Time in Representation and Design, Richmond, Surrey, Curzon Press, 1998, pp. 176-199. En el ejemplo de ciudad tradicional pueden aplicarse perfectamente terminologías biológicas: arterias, capilares, alvéolos, etc. 
"Teilhard de Chardin comparaba esta creciente complejidad (que asimismo experimentamos cuando vamos del pueblo a la ciudad) con las sucesivas dobleces de un pañuelo: cada doblez duplica las capas de material, es decir, la densidad de la experiencia. Como biólogo, establecía la analogía con el ciego impulso que provoca que la vida evolucione a partir de células simples hacia formas cada vez más complejas: un movimiento tan compulsivo como irreversible. Se trata de una curiosa intuición que probablemente explique no sólo por qué los emigrantes van del pueblo a la ciudad, sino también (y más importante) por qué, habiendo experimentado la degradación física en su nueva vida, siguen sin volver al pueblo". ${ }^{12}$

Los pliegues de la tela sobre sí misma densifican la trama y acentúan una complejidad (fig. 1) en la que se encuentran inmersos los habitantes de la metrópoli, y a la que se incorporan los que migran a ella. La primera secuencia de la película Sri 420,



Fig. 1. Charles Correa, Increasing complexity [Ilustración tomada de CORREA, Charles, The New Landscape. Urbanisation in the Third World, Singapore, Concept Media / Butterworth Architecture, 1989, p. 77]. dirigida por Raj Kapoor (R.K. Films, India, 1955) se inicia con un vagabundo que llega cantando a la ciudad de Bombay con el optimismo de saberse integrado en una inmensa pluralidad $^{13}$; aunque esa misma densidad provocaba parálisis a otra persona llegada de fuera: el cura protagonista de La Ciudad de la Alegría era incapaz de desplegar su plegaria ante la avalancha de impresiones y experiencias vividas en el slum (de Calcuta en este caso) en un corto período de tiempo $^{14}$. Podríamos adentrarnos, llegado este punto, en el mundo de las barriadas ilegales o slums que crecen en los márgenes de las ciudades indias (como de otras partes del mundo), donde se experimenta esa condensación extrema y degradación física a la que se refiere Charles Correa. En uno de ellos - el más famoso de todos, conocido como Dharavi - vive Rama Mahoma Thomas, protagonista de la novela $Q \& A$, justo en el momento en que es escogido para participar en el concurso televisivo que da nombre a la obra ${ }^{15}$. Dentro de la lógica narrativa que propone el autor, Vikas Swarup, nos llega a parecer completamente verosímil que un adolescente prácticamente analfabeto pueda llegar a conocer las respuestas de las doce preguntas que otorgan el premio de mil millones de rupias. No se trata de que

12 CORREA, Charles, The New Landscape. Urbanisation in the Third World, Singapore, Concept Media / Butterworth Architecture, 1989, pp. 76-77. La versión en castellano del capítulo "Great City... Terrible Place" citada aquí procede de CORREA, Charles, Un lugar a la sombra, Madrid, Fundación Caja de Arquitectos, 2008, p. 35.

13 KAPUR, Geeta y RAJADHYAKSHA, Ashish (2001), op. cit., p. 18.

14 LAPIERRE, Dominique, La Ciudad de la Alegría, Barcelona, Seix Barral, 2009 (1985), p. 79.

15 Versión en castellano: SWARUP, Vikas, ¿Quién quiere ser millonario?, Barcelona, Anagrama, 2009 (2005). 
una mera cuestión de probabilidad o suerte hayan favorecido a Rama; simplemente conocía las respuestas porque el flujo de situaciones de la calle estaban condensadas de algún modo en él, que había experimentado en sus propias carnes todo tipo de vivencias en torno a cada una de las preguntas del concurso. La ciudad, y en especial el suburbio donde vive, son la expresión condensada del universo, y el conocimiento de los entresijos de ese microcosmos es lo que permite que el chico pueda acertar las respuestas codificadas para un mundo más grande que el que habita. Es ahí, según Ángel González, 'en su expresión concentrada e intensa de 'lo grande', donde se juegan las condiciones y avatares de nuestra presencia física en un mundo cada vez mayor y más fuera de medida" ${ }^{\prime 16}$. La inmensa sucesión de casitas de cartón de la obra de la artista Hema Upadhyay (Baroda, 1972) titulada Dream a Wish, Wish a Dream (2004-2006, instalación de planchas metálicas y cartulinas pintadas, 457 x 762 x $60 \mathrm{~cm}$, col. Claude Berri, París), es la viva imagen en miniatura de ese macrocosmos. Upadhyay ha modelado cada una de las casitas de Dharavi - o de cualquier otro slum - recreándose en el acto mismo de doblar, definiendo cada estructura en cartulina de manera individual, simplemente porque forman parte de ese universo en expansión (fig. 2).

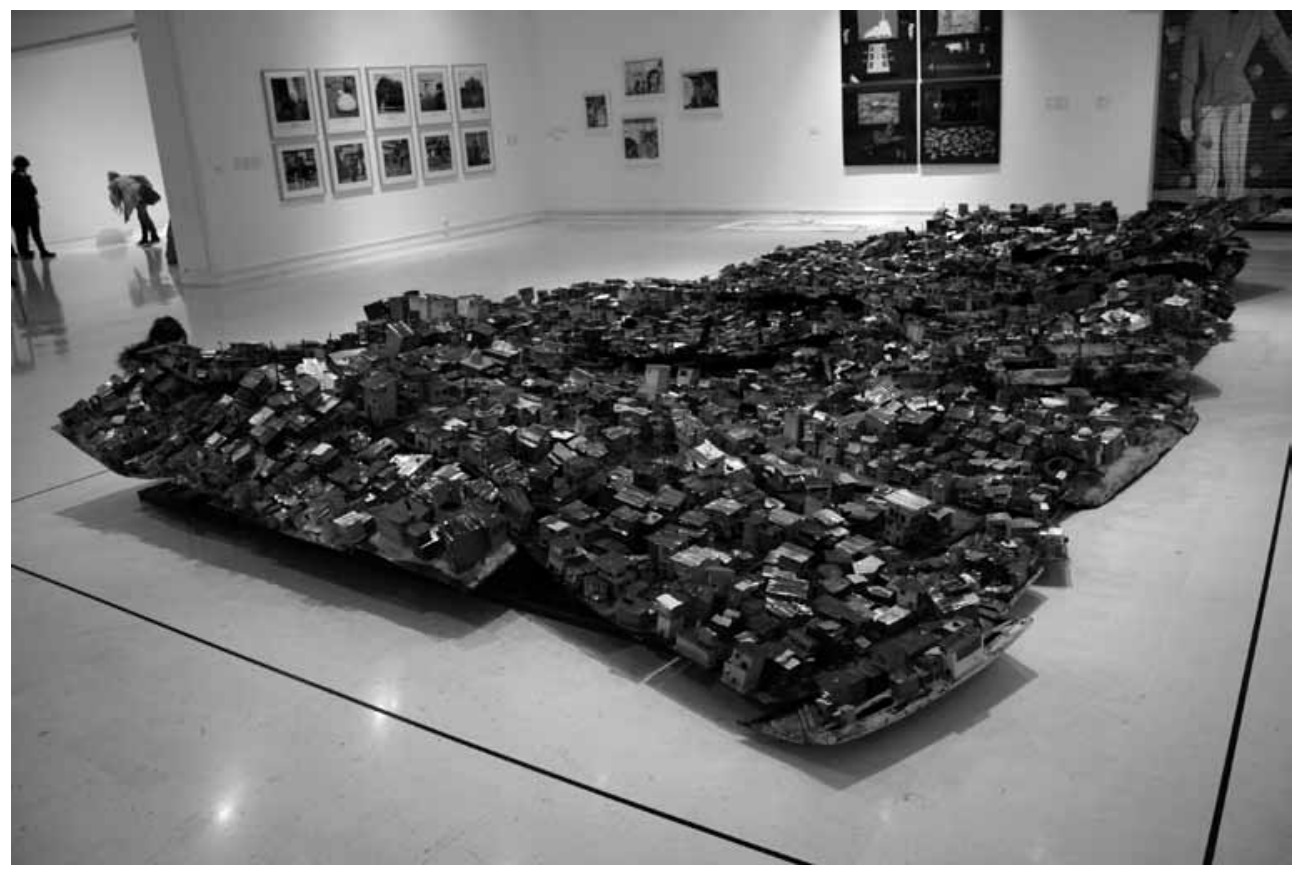

Fig. 2. Hema Upadhyay, Dream a Wish, Wish a Dream, 2004-2006, instalación de planchas metálicas y cartulinas pintadas, 457 × $762 \times 60 \mathrm{~cm}$, col. Claude Berri, París

${ }^{16}$ GONZÁLEZ GARCÍA, Ángel, "Casitas", en Pintar sin tener ni idea y otros ensayos sobre arte, Madrid, Lampreave y Millán, 2007, p. 244. 
Muchas de las barriadas chabolistas de Bombay fueron en origen poblados de pescadores, y en efecto, la ciudad de Dream a Wish parece erigirse sobre la trama de una red que flota sobre la marea. Ya hemos comentado cómo la ciudad de Bombay ha crecido literalmente sobre el mar; desde el principio estableció una relación especial con el agua, que hoy se reparte entre la carencia (de agua potable, que debe importarse de la tierra firme de Maharastra) y el exceso (del siempre desbordante monzón anual). Dharavi ve pasar de largo el agua que fluye a través de los grandes colectores herméticos que la atraviesan para irrigar a toda la ciudad de Bombay, al mismo tiempo que se inunda irremediablemente todas las temporadas de lluvia ${ }^{17}$. El agua canalizada huye, las mareas se quedan, especialmente las mareas humanas, que huyen de un medio rural con bajas posibilidades en busca de la ciudad de los sueños y los deseos. Una ciudad ilusoria, pues se extiende irrefrenablemente con las ilusiones de esas personas que llegan a la gran urbe, y que Hema Upadhyay da forma, doblez a doblez, en cada una de las casas, en un trabajo de papiroflexia infinita, cuya extensión podría no tener fin, porque, volviendo a la idea de ciudad cinética de Rahul Mehrotra, se trata de "una construcción tridimensional de desarrollo progresivo"18.

La prioridad de las personas que se incorporan a este mundo desde el medio rural no es la casa, sino el trabajo ${ }^{19}$; es decir: lo cinético (la acción y el movimiento) domina sobre la posesión de un lugar estable. De ahí que los slums suelan ubicarse en las proximidades de las vías de tren, que ponen en contacto la periferia de las ciudades con los centros urbanos, donde se encuentran los puestos de trabajo. No obstante, las dificultades de transporte en hora punta en una ciudad como Bombay explican también el hecho de que las calles céntricas posean un alto nivel de funcionalidad: por el día son ocupadas por transeúntes, vendedores callejeros o vehículos, y por las noches actúan de dormitorio masivo para esas personas que buscan estar cerca de los núcleos de generación de empleo. Charles Correa propuso ya en los años sesenta una solución para mejorar el espacio de descanso en esas condiciones: mediante un sistema de bisagras, una plataforma plana de dos por dos metros se despliega en cuatro espacios aislados del suelo y separados entre sí por cuatro paneles formando una cruz, totalmente abiertos a la intemperie, para poder acoger a cuatro personas durante la noche. Plegándolo de nuevo podrá volver a cumplir su función diurna, dar cabida a vendedores ambulantes ${ }^{20}$. El arquitecto Balkrishna V. Doshi (Pune, 1927) incide también en esa idea de multifuncionalidad, muy válida para las altas densidades en que nos movemos:

17 PRAKASH, Gyan, "Mumbai. The Modern City in Ruins", en HUYSSEN, Andreas (2008), op. cit., pp. 181-182.

18 MEHROTRA, Rahul (2008), op. cit., p. 206.

19 GROVER, Satish, Buiding beyond borders. Story of Contemporary Indian Architecture, New Delhi, National Book Trust, 1995, p. 45 y CORREA, Charles (1989), op. cit., pp. 16-17.

${ }^{20}$ CORREA, Charles, Housing \& Urbanisation, Bombay, The Urban Design Research Institute, 1999 , pp. 130-131. 
"La idea de unas escaleras que sólo cumplen la función de movimiento, de una ventana que sólo sirve para dar luz y ventilar, o un tejado que sólo ofrece refugio del clima, es ajena a nuestra cultura y no puede satisfacer la diversidad de necesidades de los distintos grupos. Una escalera puede servir para varias cosas: un lugar para sentarse o, si es grande, tal vez para dormir"21.

Las condiciones climáticas de Bombay permiten las actividades que se acaban de describir durante prácticamente todo el año, a excepción de la estación de lluvias. Como dice Suketu Mehta, "cada monzón es un asalto sobre Bombay. La lluvia torrencial es un árbitro severo y cruel sobre los principios básicos de la ingeniería. Lo que no puede hacer el ayuntamiento lo hace la lluvia: derriba estructuras poco estables"22. La obra Where the Bees Suck, There Suck I (Hema Upadhyay, 2009, instalación con pala excavadora y cartulinas pintadas, medidas variables, galería Studio La Città, Verona) ahonda en esa fragilidad. En palabras de Rama Mahoma Thomas, habitante imaginario de ese suburbio, "todas las casas de Dharavi son "construcciones ilegales", y pueden ser demolidas en cualquier momento"23. Bien el agua o bien los poderes públicos (en nombre de ese supuesto orden urbanístico) pueden llevarse por delante esas pequeñas estructuras, sin previo aviso ${ }^{24}$; motivo por el cual los habitantes de esas viviendas no invierten dinero en mejorarlas, dado que no perciben ninguna seguridad ni sensación de perdurabilidad. Siguen usando materiales de desecho, como planchas metálicas, trozos de madera, lonas, telas, etc., que podrán ser reutilizados en otro lugar por otras personas en caso de ser derribados por las máquinas. Al mismo tiempo, el carácter de los materiales y la facilidad de su reaprovechamiento constructivo provocan que este tipo de asentamientos no deje ruinas ni otro rastro de su existencia. La pala excavadora de la instalación Where the Bees Suck no hace otra cosa que trasladar los montones de casitas de un lugar a otro. Los slums, de este modo, no dejan registros para la Historia; simplemente, sus múltiples historias se trasladan a otro lugar; son lugares eventuales, flotantes, que no se ajustan a las categorías que establecía Marc Augé en su famoso ensayo ${ }^{25}$.

Viendo el ritmo de propagación de las casitas de Hema Upadhyay, a pesar de todas las amenazas externas, era lógico que tarde o temprano acabaran cubriéndolo

21 DOSHI, Balkrishna, "Social Institutions and a sense of place", en AMEEN, Farooq, Contemporary Architecture and City Form. The South Asian Paradigm, Mumbai, Marg Publications, 1997, p. 20.

22 MEHTA, Suketu (2006), op. cit., p. 161.

23 SWARUP, Vikas (2009), op. cit., p. 155.

${ }^{24}$ La instalación de Hema Upadhyay acentúa el contraste entre las casitas y los poderes casi sobrenaturales que las acechan. El título de la obra, tomado de la última canción entonada por el espíritu Ariel en $L a$ Tempestad, de William Shakespeare, preludia el momento de liberación de los encantamientos producidos por la magia del protagonista, Próspero. Podríamos interpretar la obra como un deseo de liberación del destino al que se supone que están abocadas las viviendas ilegales de los slums. Ver SHAKESPEARE, William, La tempestad (Edición bilingüe del Instituto Shakespeare dirigida por Manuel Ángel Conejero Dionís-Bayer), Madrid, Cátedra, 2007, pp. 386-387.

25 AUGÉ, Marc, Los «no lugares». Espacios del anonimato. Una antropología de la Sobremodernidad, Barcelona, Gedisa, 2000 (1992). 
todo. Es la evolución lógica de la serie empezada por Upadhyay hacia 2004 y que se completa - por el momento - con instalaciones como la titulada 8 feet $x 12$ feet (2009, cartulinas pintadas, plástico y planchas de aluminio, galería Chemould Prescott, Bombay), entre otras. En este caso, las pequeñas casas de materiales de desecho conviven con los bloques de edificios (como ocurre realmente en algunas zonas de suburbios como Dharavi), todos ellos reproducidos en cartulinas de colores y dispuestos por las paredes y el techo de una habitación (fig. 3). De este modo, el microcosmos de dobleces ha llegado a un punto de compactación máxima, cerrándose sobre sí mismo en una especie de caja. Cuando toda esa cantidad de energía y capacidad de replicación queda encerrada puede llegar a implosionar, como lleva alertando Charles Correa desde hace décadas: Bombay aumenta su complejidad humana y potencia su carácter de gran ciudad al mismo tiempo que se hace verdaderamente inhabitable como entorno físico ${ }^{26}$.

Si el beneficio de vivir en la ciudad lo constituye la densidad de relaciones humanas que en ella se establecen y el problema surge a la hora de plantear el lugar del habitar, especialmente para las personas con menos recursos, la pregunta surge inmediatamente: ¿Cómo conciliar las altas densidades con un medio urbano sostenible para la vida? Es la misma cuestión que se han planteado desde hace décadas los arquitectos que hemos ido nombrando a lo largo del presente texto.

Cuando Charles Correa citaba a Teilhard de Chardin, aplicando sus ideas a las metrópolis indias contemporáneas,



Fig. 3. Hema Upadhyay, 8 feet $x 12$ feet, 2009, cartulinas pintadas, plástico y planchas de aluminio, Galería Chemould Prescott. estaba desarrollando una interesante intuición biológica del urbanismo que le acerca enormemente a un arquitecto de gran importancia para la India independiente, que no podía ser otro que Le Corbusier (1887-1965). A la vuelta de uno de los viajes que realizó a India (1954) para la planificación y construcción de Chandigarh, Le Corbusier envió una carta a uno de

${ }^{26}$ CORREA, Charles (2008), op. cit., p. 35. 
sus colaboradores, mostrándole su especial interés por las teorías sobre la planetización humana de Teilhard de Chardin, ideas que calificaba de "definitivas" en su apreciación del concepto de comunidad ${ }^{27}$. Con toda seguridad, ciertos fragmentos destacados de las teorías del paleontólogo francés vinieron a reforzar las ideas que Le Corbusier ya venía manejando, especialmente las que incidían en una densificación creciente tendente a la unidad ${ }^{28}$, que se adaptan sin demasiados problemas a la Unité d'Habitation (1947-53, Marsella), modelo ideal que eventualmente podría replicarse para obtener una comunidad más grande. La clave de la interpretación de Correa se aleja radicalmente de Le Corbusier en este punto: "La respuesta se encuentra en el pluralismo, no en la clonación" 29 . La conexión biológica del urbanismo (de la que Le Corbusier, por otra parte, era partidario ${ }^{30}$ ) no podría ser otra que la de favorecer la vida, y aunque suene sencillo, debería tenerse claro que "la vida sólo es posible en los sistemas lejos del equilibrio. La estabilidad equivale a la destrucción del sistema [...]. La quietud y la inmutabilidad parecen lo más ajeno a la vida" 31 .

Una de las propuestas urbanísticas más relevantes de Charles Correa para la vivienda de bajo coste, muy próxima a los ritmos biológicos que se reclaman aquí, es el Belapur Housing o Artiste's Village (Belapur, Nuevo Bombay, 1983-1986), tomado como modelo en numerosas ocasiones para ilustrar los conceptos de "vivienda progresiva", y en el que veremos cómo se pretenden alcanzar altas densidades respetando escrupulosamente los espacios jerárquicos de relación social. Lo que plantea Correa es un modelo de desarrollo progresivo (casi podríamos relacionarlo con la replicación celular) basado en estructuras vecinales simples que, siguiendo la base orgánica del principio de desdoblamiento, acaban configurando una comunidad compleja (fig. 4). La clave del sistema se encuentra en la progresión de espacios comunes: 1) el patio privado de la casa; 2) el patio vecinal de primer nivel, de 8 × 8 metros, que pone en contacto a un máximo de siete familias; 3 ) el espacio de encuentro vecinal de segundo nivel, de 12 × 12 metros, donde se puede encon-

27 Sobre el interés de Le Corbusier por Teilhard de Chardin, y su posible relación con los proyectos de viviendas del arquitecto, ver SAMUEL, Flora, "Le Corbusier, Teilhard de Chardin and "The Planetisation of Mankind', en The Journal of Architecture, vol. 4, n. 2, 1999, pp. 149-165.

28 TEILHARD DE CHARDIN, Pierre, El porvenir del hombre, Madrid, Taurus, 1967 (1959). Podríamos citar frases como: "las partículas humanas no sólo se multiplican más cada día, sino que, por reacción a sus mutuos roces, desarrollan en torno a sí, automáticamente, una madeja cada vez más densa de conexiones económicas y sociales [...]. ¿No es evidente que una sola dirección permanece abierta al movimiento que nos arrastra: la de una unificación siempre creciente?”, p. 159.

${ }^{29}$ CORREA, Charles, "The Challenge of Humanizing Large Housing Projects. Introduction", en BENTLEY SEVCENKO, Margaret (ed.), Large Housing Projects. Design, Technology, and Logistics (Designing in Islamic Cultures 5), Cambridge, Massachusetts, The Aga Khan Program for Islamic Architecture, 1985, p. 2

30 LE CORBUSIER, Le Corbusier Sketchbooks. 2. 1950-1954, London, Thames and Hudson, 1981. El dibujo número 880 combina distintos dibujos de animales junto al Modulor y una frase en la parte superior: "El urbanismo es una organización biológica".

31 AIZPÚN, Teresa, Gramática de la vida. La ética como creatividad, Madrid, Endymion, 2009, pp. 46-47. 

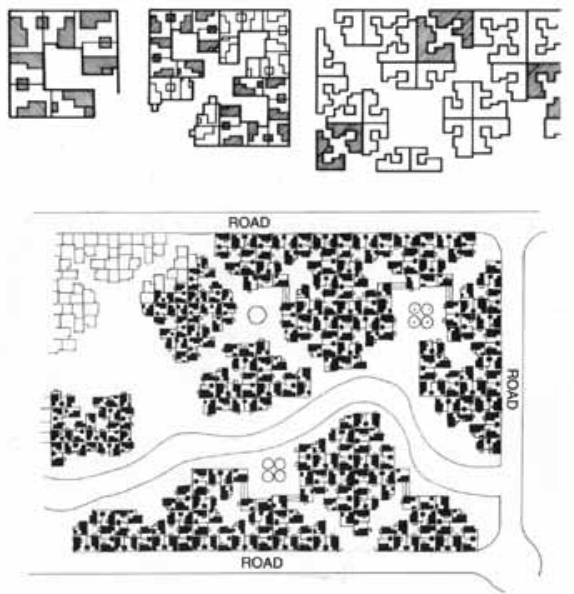

Fig. 4. Charles Correa, Belapur Housing (Belapur, Nuevo Bombay, 1983-1986). Plano de los módulos de crecimiento progresivo y del complejo general [Tomado de BHATT, Vikram y SCRIVER, Peter, Contemporary Indian Architecture. After the Masters, Ahmedabad, Mapin Publishing, 1990, p. 103]. trar el pozo o la fuente públicas, y 4) la plaza principal, también conocida como maidan, que es el centro de la comunidad, con unos 20 x 20 metros $^{32}$. Mediante la organización de espacios públicos en jerarquía se pone en práctica un sistema con múltiples corazones, en sustitución del habitual modelo de arteria (vía principal o secundaria) en torno a la que se disponen los hogares, en hilera.

"Identificar este sistema jerárquico es, por supuesto, el primer paso esencial hacia la provisión de viviendas viables. [...] No comprenderlo explica por qué tantos intentos de viviendas de bajo coste son percibidos sólo como una cuestión simplista de acumulación de tantas unidades (a modo de celdas) como quepan en un lugar concreto, sin ninguna preocupación por el resto de espacios implicados en esta jerarquía" ${ }^{33}$.

A este respecto, la obra Hovering over the local landscape (2008, acuarela, pastel y lápiz sobre papel, 213 x $137 \mathrm{~cm}$, Vadhera Art Gallery, Nueva Delhi) del artista Zakkir Hussain (1970, Chandiroor, Kerala) muestra de qué modo la mera clonación que omite la progresión de espacios, y que por tanto forma un bloque impenetrable, puede llegar a "traer de cabeza a sus habitantes" (fig. 5).

Pero volviendo a la buscada organicidad y humanización de los proyectos de viviendas de alta densidad, hay un punto fundamental en el que debemos detenernos antes de finalizar: la revisión del papel del arquitecto. El proyecto de Charles Correa en Belapur (aunque podríamos citar otros ejemplos del mismo autor), que es comparable en muchos aspectos al Aranya Low-Cost Housing ${ }^{34}$ (Indore, 1983-86) de Balkrishna Doshi, propone una organización de los solares y los espacios comunes, cuyos límites (como si de un código genético se tratara) se mantendrán invariables a lo largo del tiempo, al tiempo que los equipa con una arquitectura a modo de propuesta, con la posibilidad siempre abierta de que el propietario la adapte a sus necesidades y gustos (o incluso la derribe para elevar su diseño particular).

${ }^{32}$ CORREA, Charles (1989), op. cit., pp. 32-34.

33 Ibid., p. 36.

${ }^{34}$ Una de las principales diferencias entre el proyecto de Aranya (Doshi) y el de Belapur (Correa) radica en la incorporación del tráfico rodado en las vías principales. En Belapur es externo al complejo, mientras que en el proyecto de Doshi, las plazas públicas son ensanchamientos de las vías de comunicación. Ver STEELE, James, The Complete Architecture of Balkrishna Doshi. Rethinking Modernism for the Developing World, London, Thames \& Hudson, 1998, pp. 114-129. 
De este modo, el papel del arquitecto se condensa de manera absoluta en los espacios vacíos, y de modo relativo o temporal en las construcciones tridimensionales que a menudo suelen representarles ${ }^{35}$. Mediante esta estrategia se pretende conseguir que las casas puedan crecer a lo largo del tiempo, a medida que sus dueños lo vayan necesitando, en función de las cuestiones económicas, laborales o familiares. Por tanto, el carácter progresivo del crecimiento urbano, si tomamos como ejemplo estos proyectos, se expande primero en el espacio y luego se desarrolla en el tiempo según los patrones espaciales fijados previamente, con la intención de favorecer todas las condiciones de vida y de relación social, aunque obviamente dentro de una escala local. Las grandes ciudades como Bombay seguirán constituyendo un macrocosmos infinitamente más complejo. Allí, en palabras de Rahul Mehrotra, "la comprensión de



Fig. 5. Zakkir Hussain, Hovering over the local landscape, 2008, acuarela, pastel y lápiz sobre papel, $213 \mathrm{x}$ $137 \mathrm{~cm}$, Vadhera Art Gallery. que el significado cultural evoluciona, retará tanto como clarificará el rol del arquitecto como defensor del cambio [...]; un rol que pueda establecer relación con la Ciudad Cinética tanto como con la Estática, en términos igualitarios" ${ }^{\prime 36}$. Y donde sería deseable un mayor equilibrio entre la densidad de las vivencias urbanas y el medio físico que debe favorecerlas.

35 En más de una ocasión se ha recurrido al término "Arquitectura sin arquitectos" en este tipo de contextos. Ver BHATT, Vikram y SCRIVER, Peter, Contemporary Indian Architecture. After the Masters, Ahmedabad, Mapin Publishing, 1990, pp. 93-95.

36 MEHROTRA, Rahul (2008), op. cit., p. 211. 